\title{
Dual targeting of FGFR3 and the ERBB receptor family or AXL enhances the efficacy of FGFR inhibitors in FGFR3 fusion-driven bladder cancer
}

\section{David Lau}

Olivia Newton John Cancer Research Institute

\section{Margeaux Hodgson-Garms}

Olivia Newton John Cancer Research Institute

\section{Austen Lavis}

Olivia Newton John Cancer Research Institute

lan Luk

Olivia Newton John Cancer Research Institute

\section{Laura Jenkins}

Olivia Newton John Cancer Research Institute

Natalia Vukelic

Olivia Newton John Cancer Research Institute

Paul loannidis

Olivia Newton John Cancer Research Institute

John Mariadason ( $\nabla$ john.mariadason@onjcri.org.au )

Olivia Newton John Cancer Research Institute

\section{Andrew Weickhardt}

Olivia Newton John Cancer Research Institute

\section{Research Article}

Keywords: Bladder cancer, FGFR3, EGFR, ERBB2, ERBB3, AXL, Targeted therapy, Acquired resistance

Posted Date: January 19th, 2021

DOI: https://doi.org/10.21203/rs.3.rs-142420/v1

License: (c) (i) This work is licensed under a Creative Commons Attribution 4.0 International License.

Read Full License 


\section{Abstract}

\section{Background}

Mutations and fusions in Fibroblast Growth Factor Receptor 3 (FGFR3) occur in 10-20\% of metastatic urothelial carcinomas, and can confer sensitivity to FGFR inhibitors such as BGJ398 and erdafitinib. However, responses to these agents are often short-lived due to the development of acquired resistance. The objective of this study was to identify mechanisms of acquired resistance to FGFR inhibitors in two previously uncharacterized bladder cancer cell lines harbouring FGFR3 fusions, and assess rational combination therapies to enhance their activity.

\section{Methods}

Acquired resistance to FGFR inhibitors was generated in two FGFR3 fusion harbouring cell lines, SW780 (FGFR3-BAIAP2L 1 fusion) and RT4 (FGFR3-TACC3 fusion), by either long-term exposure to increasing concentrations of BGJ398 or sustained exposure to high concentrations of drug. Alterations in levels of key cell signalling regulators was assessed in resistant lines by phospho-RTK arrays and immunoblotting. Synergy between BGJ398 and alternate targeted therapies was explored using cell viability and apoptosis assays.

\section{Results}

Acquired resistance to BGJ398 in SW780 and RT4 cells was associated with increased expression of members of ERBB family of receptor tyrosine kinases and PAXL. Combination treatment of resistant cells with an FGFR inhibitor and either a pan-ERBB or an AXL inhibitor overcame this resistance. We also noted rapid reactivation of pERK in parental FGFR3 fusion-driven lines within 4-72 hours of BGJ398 treatment, with concomitant increase in pERBB3. Up-front combination treatment with BGJ398 and the pan-ERBB inhibitor AZD8931 delayed the reactivation of $\mathrm{pERK}$, and induced a synergistic inhibition of cell viability and a concomitant increase in apoptosis.

\section{Conclusions}

We identify increased activation of AXL and ERBB family receptors as mechanisms of resistance to FGFR inhibition. Our findings suggest that upfront combination treatment with FGFR and pan-ERBB inhibitors warrants further investigation for FGFR3-fusion harbouring bladder cancers.

\section{Introduction}

Urothelial bladder cancer is responsible for approximately 150,000 deaths per year worldwide, and the median survival of patients with metastatic disease is approximately 18 months $(1,2)$. Fibroblast Growth Factor Receptor 3 (FGFR3) is an attractive target in bladder cancer given the $10-30 \%$ prevalence of $F G F R 3$ aberrations (activating mutations or aberrant gene fusions) in these tumours, and their preclinical sensitivity to FGFR-targeted therapy (3). 
FGFR3 aberrations lead to oncogenic signalling through the MAPK and PI3K pathways. Activating mutations in FGFR3 occur in $10-20 \%$ of muscle-invasive bladder cancers (4-9) and a higher proportion in superficial and upper tract urothelial cancer (10). These mutations cluster in hotspots within exons 7, 10, and 15 of the FGFR3 gene, with 5 mutations, R248C, S249C, G372C, Y375C, and K652E accounting for greater than $90 \%$ of all mutations $(5,8,11,12)$. These mutations induce ligand-independent receptor dimerization, transactivation, and constitutive activation of downstream signalling (13-15).

A smaller proportion of bladder cancers (3-6\%) have chromosomal translocations involving FGFR3 which generate oncogenic FGFR3 fusion proteins $(4,16,17)$. These fusion proteins comprise of amino acids 1760 of FGFR3 (which include the kinase domain) fused in-frame to either transforming acid coiled-coil 3 (TACC3) or BAI-Associated Protein 2-Like-1 (BAIAP2L 1) (5), and form overexpressed, permanently dimerized inclusion bodies in the cytosol that do not undergo lysosomal degradation, and are not susceptible to feedback inhibition (17). The FGFR3 component of the fusion gene is identical, with a conserved break-point lacking only the final exon (exon 19). Expression of these fusion proteins in normal human urothelial cells has been shown to induce mitogenic activation of the MAPK pathway (18).

Preclinical studies have demonstrated that human bladder cancer cell lines with FGFR3 mutations and fusions are sensitive to FGFR inhibitors such as BGJ398 (Novartis), PD173074 (Pfizer) and erdafitinib (JNJ-42756493, Janssen) $(17,19-22)$, forming the basis for clinical trials of FGFR inhibitors in patients with metastatic urothelial cancer. Results from phase I and II trials of BGJ398 and erdafitinib in this population reported response rates of $25-40 \%$, however median progression-free survival was only 3.7-5.5 months. $(23,24)$

Several studies have investigated the mechanisms driving inherent and acquired resistance to FGFR inhibitors. However, studies in models of FGFR3-fusion harbouring bladder cancer lines have so far been limited to a single cell line, RT-112, which harbours a FGFR3-TACC3 fusion as well as an amplification (25-27). Mechanisms of resistance described in this model include epithelial-to-mesenchymal transition (25), activation of EGFR (26), ERBB2 and ERBB3 (25), and increased activation of AKT (27). However, whether these mechanisms extends to other FGFR3-fusion driven bladder cancer cell line is unknown.

To address this, we undertook this preclinical study to investigate potential mechanisms of resistance to FGFR inhibitors in two previously uncharacterized bladder cell line models of FGFR3-fusions, RT4 (FGFR3-TACC3 fusion) and SW780 (FGFR3-BAIP2L 1 fusion). We observed an increase in PAXL and members of the ERBB receptor family in cells with acquired resistance to FGFR inhibition, and demonstrate that combination treatment with inhibitors of PAXL or the ERBB receptor family, can resensitize these lines to FGFR inhibitors. We also demonstrate that rapid reactivation of pERK in FGFR3driven cell lines following FGFR inhibitor treatment can be overcome by ERBB receptor blockade, suggesting treatment with this combination from the onset may represent a more effective approach for treating FGFR3-fusion driven bladder cancers.

\section{Methods}




\section{Cell lines, culture and reagents}

The urothelial carcinoma cell lines SW780 (FGFR3-BAIP2L 1 fusion) and RT4 (FGFR3-TACC3 fusion) were obtained from the American Type Culture Collection (ATCC). Cells were maintained in Dulbecco's Modified Eagle Medium (DMEM/F-12, plus glutamine and sodium bicarbonate (Invitrogen, Carlsbad, CA, USA) supplemented with 1\% GlutaMax, 1\% HEPES, 1\% Penicillin/Streptomycin, and 10\% Fetal Bovine Serum (Invitrogen), and incubated at $37^{\circ} \mathrm{C}$ in $5 \%$ Carbon Dioxide. BGJ398 was obtained from Novartis (Basel, Switzerland). AZD8931, R428 and erdafitinib were purchased from Selleck Chemicals (Houston, TX, USA).

\section{Establishment of drug resistant cell lines}

Resistance to BGJ398 in SW780 and RT4 bladder cancer cell lines was established by either gradual dose escalation of drug (referred to as SW780 RD and RT4 RD) or by sustained exposure to $1 \mathrm{mM} \mathrm{BGJ398}$ (labelled SW780 RS and RT4 RS). Gradual dose escalation (RD cell lines) began with treatment of $3 \mathrm{nM}$ BGJ398 with stepwise concentration escalation until cells were able to proliferate in $1 \mu \mathrm{M}$ of drug. Comparatively, SW780 RS and RT4 RS cell lines were continuously maintained in $1 \mu \mathrm{M}$ BGJ398, with fresh drug added each time the cells were passaged. Parental cell lines were passaged in parallel in equivalent concentrations of DMSO. Parental and resistant cells were regularly assessed for Mycoplasma contamination and the authenticity of the cell lines verified using the Promega StemElite ID System.

\section{Cell viability and apoptosis assays}

Cell viability was measured using either the CellTitre-Glo luminescent cell viability assay (Promega, Madison, WI, USA) or the MTS assay (Promega). For CellTitre-Glo assays, cells were seeded in 96 flat bottom well plates at a density of 1500-5000 cells per well, then treated the following day with drug for 72 hours. Luminescence was measured using a SpectraMax L Microplate Reader (Molecular Devices, Sunnyvale, CA, USA) and compared to DMSO treated cells. MTS assays were performed using the same protocol, except cell viability was assessed after $72 \mathrm{~h}$ using the CellTiter 960 Aqueous MTS reagent (Promega) as per manufacturer's instructions, and measurement of absorbance at $490 \mathrm{~nm}$ (MTS) and $630 \mathrm{~nm}$ (background absorbance) using a SPECTROstar Nano microplate reader (BMG labtech, Germany).

Apoptosis was assessed following 72 hours of drug treatment by Propidium lodide staining as described previously (28), followed by FACS analysis using a BD FACS Canto II flow cytometer (BD Biosciences San Jose, CA). The percentage of apoptotic cells was determined by calculating the percentage of cells with a sub-diploid DNA content using the FLOWJO software V10.0 (FlowJO LLC, Ashland, OR, USA).

\section{Phospho-receptor tyrosine kinase (RTK) arrays}

RT4-RS and SW780-RS cells were cultured in fresh media without drug for 24 hours prior to collection to negate effects induced by acute drug exposure. Control and resistant lines were then lysed in Radio immunoprecipitation assay buffer (Sigma-Aldrich, St Louis, MO, USA) containing complete Protease Inhibitor Cocktail Tablets (Roche, Basel, Switzerland) and PhosSTOP (Roche). $200 \mathrm{mg}$ of lysate was then 
incubated with human Phospho-RTK Arrays (ARY001B, R\&D Systems, Minneapolis, MN, USA) as per manufacturer's instructions, and blots were read using the ChemiDoc X Imaging System (Bio-Rad, Hercules, CA, USA).

\section{Immunoblotting}

Protein lysates were prepared as above, denatured using 10x NuPage Sample Reducing Agent (ThermoFisher, Waltham, MA, USA), and run on NuPAGE Novex 4-12\% Bis-Tris precast gels (Invitrogen) in MES (2-( $N$-morpholino)ethanesulfonic acid) buffer (ThermoFisher). Proteins were transferred using the iBlot ${ }^{\circledR}$ Dry Blotting System (Invitrogen) and signal detected using the Li-Cor ${ }^{\circledR}$ Odyssey Infrared Imager (Li-Cor, Lincoln, NE, USA). The following antibodies were obtained from Cell Signaling Technologies (Danvers, MA, USA): pERK p44/42 MAPK T202/Y204 (9106); t-ERK p44/42 MAPK (9107), p-ERBB3 (2842S), ERBB3 (4754S), EGFR (2232), pEGFR Tyr1068 (D7A5), AXL (8661) and p-AXL (5453). Anti- btubulin (ab6046) was obtained from Abcam (Cambridge, UK).

\section{Statistical analysis}

Statistical analyses were performed using Prism v5 (GraphPad Software, La Jolla, CA, USA). Data shown is mean $\pm S E M$ from 3 technical replicates from a representative experiment unless stated otherwise. Biological replicates were performed for the majority of experiments and are stated in the figure legends. Groups were compared using parametric unpaired Student's t-test with Welch's correction. $P$-values $\leq 0.05$ were considered to be statistically significant. Synergy was determined using the synergy ratio formula

$$
S R=\frac{\% \text { Survival }[\text { Combination }]}{\% \text { Survival }[\text { drug } 1] \times \% \text { Survival }[\text { drug } 2]}
$$

whereby, SR $<0.8$ is considered synergistic, SR 0.8

-1.2 is considered additive, and SR $>1.2$ considered antagonistic.

\section{Results}

\section{Generation of bladder cancer cell lines with acquired resistance to FGFR inhibitors}

SW780 and RT4 cell lines with acquired resistance to the FGFR inhibitor BGJ398 were generated using two approaches. SW780-RD and RT4-RD cells were generated by stepwise exposure to increasing concentrations of BGJ398 for 3 months culminating at $1 \mathrm{mM}$, a dose which is approximately 2-fold higher than the clinically reported Cmax of BGJ398 (29). Comparatively, SW780-RS and RT4-RS were generated by continuous culture of the lines at $1 \mu \mathrm{M}$ for 3 months. All resistant cell lines were subsequently maintained at $1 \mu \mathrm{M} \mathrm{BGJ398}$. Response of the two sets of cell lines to BGJ398 was subsequently assessed by CellTitre-Glo assay which confirmed that SW780-RS, SW780-RD, RT4-RS and RT4-RD were significantly more resistant to BGJ398 compared to matched parental control cell lines that had been cultured in parallel (Figure 1A, B). The SW780-RS line was also found to be cross-resistant to a second FGFR inhibitor, erdafitinib (Figure 1C). 
To investigate the mechanistic basis for acquired resistance to BGJ398, the phosphorylation status of 49 receptor tyrosine kinases (RTKs) was compared between parental and SW780-RS and RT4-RS cells using phospho-RTK arrays. This screen identified an increase in pAXL and pERBB3 in both SW780 RS and RT4 RS cell lines (Figure 2A) and increased pEGFR and pERBB2 in SW780 RS cells. These changes were confirmed by western blot, which also revealed similar changes in the SW780-RD and RT4-RD lines and an increase in pERBB2 as well as total ERBB2 in RT4-RS (Figure 2B). Notably, these analyses also revealed an increase in total $A X L$ levels, suggesting this increase may drive the increase in PAXL levels.

\section{Investigation of combination therapy targeting FGFR3 and pAXL or FGFR3 and the ERBB family in FGFR- resistant blander cancer lines}

Based on the observed increase in PAXL and members of the ERBB family, we examined the effect of combining BGJ398 with either an inhibitor of AXL kinase activity (R428, BerGenBio) or the pan-ERBB family inhibitor AZD8931 (Astra Zeneca) in FGFR inhibitor-resistant cell lines (30).

Combination treatment of SW780-RS and RT4-RS cells with BGJ398 and the AXL inhibitor R428 resulted in modest additive suppression of cell viability in SW780-RS cells and a more robust synergistic inhibition of cell viability in RT4-RS cells (Figure 3A, B). However, this combination failed to enhance the apoptosis induction in either cell line (Figure 3C, D). Comparatively, combination treatment with BGJ398 and AZD8391 synergistically inhibited cell viability in both SW780-RS and RT4-RS cells (Figure 4A, B). This combination resulted in a concomitant increase in apoptosis (Figure 4C, D).

\section{Reactivation of MAPK signalling is an early adaptive mechanism of FGFR inhibition}

While these analyses reflected mechanisms of acquired resistance associated with long-term FGFR inhibition, it is now evident that several tumours, including bladder cancers, also can rapidly adapt to targeted therapies and reactivate signalling through a variety of mechanisms $(25,31,32)$. We therefore assessed the effect of FGFR inhibition on MAPK signalling in SW780 and RT4 parental cells over 72 hours. Remarkably, while pERK levels were initially suppressed by BGJ398 at 4 hours, the magnitude of suppression gradually diminished and pERK levels rebounded to close to basal levels by 72 hours (Figure 5A, B). We next investigated whether this feedback was associated with changes in PAXL and ERRB family members. Levels of PAXL and total AXL were undetectable in untreated and treated SW780 and RT 4 cells over the 72 hour time course (not shown), demonstrating AXL levels are not rapidly induced following BGJ398 treatment. Comparatively, while minimal induction of pERBB2 or pEGFR was observed over the 72 hour time course in either cell line, robust induction of pERBB3 was observed in both cell lines within 24 hours.

Finally, we investigated whether the rapid reactivation of MAPK signalling in FGFR-fusion harbouring bladder cancer cells could be attenuated by combined treatment with BGJ398 and the pan-ERBB inhibitor, AZD8931, and whether this could enhance the growth inhibitory effect of BGJ398. Indeed, combined treatment of both RT4 and SW780 cells with BGJ398 and AZD8931 significantly attenuated the induction of pERBB3, and further suppressed pERK levels compared to the effect of either agent alone (Figure 6A). 
Furthermore, combination treatment with BGJ398 and AZD8931 synergistically inhibited cell viability in both SW780 and RT4 cells (Figure 6B). Notably, this effect was observed at a concentration of $0.1 \mathrm{mM}$ BGJ398, approximately 5-fold lower than the clinically achievable concentration of $0.5 \mathrm{mM}(29)$.

\section{Discussion}

The FGFR inhibitors BGJ398 and erdafitinib induce objective responses in 25-40\% of patients with metastatic urothelial cancer harbouring FGFR3 alterations(23, 24, 33, 34). However, the median progression-free survival in these patients is typically less than six months. These findings point to the existence of two types of resistance - inherent resistance which precludes $60-70 \%$ of patients responding at all, and acquired resistance, where tumours which initially respond invariably develop resistance. These observations are similar to the extensive clinical experience with other targeted therapies where initial responses are often followed by tumour progression after 6-12 months (35-37). To better understand the mechanisms of inherent and acquired resistance to FGFR inhibition, we undertook in vitro modelling of these processes in two previously uncharacterized FGFR3-fusion driven cell lines, SW780 (FGFR3-BAIP2L 1 fusion) and RT4 (FGFR3-TACC3 fusion).

Interestingly, a prior study reported that not all FGFR3 altered cell lines remain dependent on FGFR for growth in vitro, with some cell lines switching their dependence to ERBB family members. This is reflected in different inherent sensitivities to FGFR inhibitors (26). The basis for this switch is unknown, but may reflect the availability of ligands in the medium. Notably, both FGFR3-fusion harbouring cell lines investigated in the current study were sensitive to BGJ398, indicating they retain their dependence on FGFR signalling and are therefore suitable models to investigate mechanisms of acquired resistance.

In this study, we examined resistance mechanisms to the FGFR inhibitor BGJ398 in two contexts acquired resistance following long term exposure to BGJ398, and rapidly (4-72 hours) following BGJ398 treatment - in two previously uncharacterized models of bladder cancer cell lines harbouring FGFR3fusions. In the acquired resistance setting we identified increased expression of $\mathrm{pAXL}$ and several members of the ERBB family of RTKs. Importantly, combination treatment with either a pAXL or a panERBB inhibitor re-sensitized these lines to FGFR inhibition, indicating a direct role for these proteins in conferring resistance.

Consistent with our findings, AXL has been identified as a potential target for overcoming resistance to EGFR inhibitors in non-small cell lung cancer (38-40). Inhibition of AXL was also synergistic when used in combination with the anti-mitotic agent docetaxel in NSCLC cell lines that had developed resistance by undergoing epithelial to mesenchymal transition (EMT) (41). Although the onset of EMT was not directly examined in our study, it has been previously associated with acquired resistance to FGFR inhibitors in RT112 cells (25), although whether this directly contributes to resistance was not established. As AXL is a well-established inducer of EMT (42), inhibition of AXL may contribute to the re-sensitization of these tumours to FGFR inhibitors by reversing the EMT process. However, the design of specific AXL inhibitors has been challenging to date due to the absence of a three-dimensional structure of its kinase domain, 
with most AXL inhibitors inhibiting multiple tyrosine kinases (43). We elected to use R428 (BGB324) (BerGenBio) to target AXL due to its activity and selectivity (44). R428 has undergone a successful phase 1 trial demonstrating reasonable tolerability and activity in acute myeloid leukaemia (45), and is currently being tested in multiple clinical trials (NCT02922777, NCT02488408, NCT02424617, NCT02872259). The ongoing development of $A X L$ inhibitors raises the possibility of using these agents to overcome FGFR inhibitors' resistance.

We also observed that acquired resistance to FGFR-inhibition in FGFR3-fusion driven bladder cancer cell lines is associated with increased activation of multiple ERBB family members. This finding is consistent with observations in a different FGFR3 fusion-driven bladder cancer line, RT112, $(25,46)$, suggesting this may be a common resistance mechanism in these tumours. In RT112 cells, the authors reported increased pERBB2 and pERBB3 following long term culture with BGJ398. Resistant cells also had elevated levels of the ligands NRG1 and NRG2, which activate ERBB2/3, and provision of exogenous NRG1 or NRG2 was able to rescue parental RT-112 cells from BGJ398 induced cell death.

An important finding of the current study is the capacity of FGFR3-fusion harbouring bladder cancer cells to rapidly reactivate MAPK signalling. We demonstrate that this is associated with increased pERBB3 levels, and that $\mathrm{PERK}$ reactivation could be attenuated by combined treatment with the pan-ERBB inhibitor, AZD8931. In parallel, combined FGFR/ERBB inhibition further inhibited cell growth of FGFR3fusion driven bladder cancer lines. Notably, in RT-112 cells treated with BGJ398, pERBB2 and pERBB3 were reported to be rapidly elevated within $24 \mathrm{~h}$ (25). Furthermore, Herrera-Abreu et al also demonstrated that RT-112 cells express high basal levels of EGFR, and that EGFR-signaling is rapidly induced following BGJ398 treatment, limiting its response to BGJ398 (26). While we did not observe a rapid increase in pERBB2 or pEGFR in SW780 and RT4 cells following BGJ398 treatment, both receptors are expressed in these cells, particularly EGFR at high levels. As ERBB3 can activate signalling through hetero-dimerization with ERBB2 and EGFR (47), one possibility is that ERBB3 heterodimerizes with existing ERBB2 and EGFR to reactivate MAPK signalling in these cells. On the other hand, cross-talk between ERBB and FGFR receptors has previously been described, with ERBB3 shown to be required for maintaining FGFR2 phosphorylation and proliferation in some FGFR2-amplified gastric cancer cells (48). A further possibility therefore is that ERBB3 may cooperate with FGFR3 to reactivate MAPK/ERK signaling. While additional studies are required to define the specific mechanisms by which ERBB receptors contribute to signaling in FGFR3-fusion driven bladder cancers, our findings in the SW780 and RT4 cell line models add to the previous findings in RT112 cells to demonstrate compensatory activation of ERBB-driven signaling as a consistent mechanism of rapid adaptive resistance to FGFR inhibitors in these tumours.

Critical to preventing the emergence of these diverse resistance mechanisms is the more efficient targeting and elimination of FGFR-driven tumours from the onset of treatment. In this regard, the finding that compensatory activation of ERBB-driven signalling occurs rapidly in response to FGFR inhibitor treatment in multiple models of FGFR3-fusion harbouring bladder cancer, and our demonstration that dual targeting of FGFR and ERBB family receptors synergistically inhibits growth, suggests that dual blockade of FGFR and ERBB-driven signalling may represent a more effective treatment strategy for these 
tumours. Further preclinical work using xenograft models would be required to explore this combination's effectiveness and toxicity before clinical trials.

\section{Conclusions}

We identify increased activation of AXL and ERBB family receptors as mechanisms of resistance to FGFR inhibition in FGFR3-fusion driven bladder cancer cell lines. Our findings suggest that upfront combination treatment with FGFR and pan-ERBB inhibitors warrants further investigation for these tumours.

\section{Declarations}

Ethics approval and consent to participate: Not applicable

Consent for publication: Not applicable

Availability of data and materials: All data generated or analysed during this study are included in this published article [and its supplementary information files].

Competing interests: AW received honoraria from Novartis, Pfizer, Merck; AW advisory board Novartis, Pfizer, Ipsen, BMS, Merck. AW travel support Ipsen.

Funding: This work was supported by an Austin Medical Research Foundation grant. AW received support from Pfizer Cancer Research Grant, and Novartis (BGJ398 supplied). DL and LJJ received financial support La Trobe University (Australian Postgraduate Award) and DL funding from the Pancare/RMA Network. JM was supported by a NH\&MRC Senior Research Fellowship (1046092). The Olivia NewtonJohn Cancer Research Institute acknowledges the support of Ludwig Cancer Research, and the Operational Infrastructure Support Program of the Victorian State Government.

Authors' contributions: The study was conceived by AJW, DL and JMM. Experiments and data analysis were performed by DL, MGH, AL, IL, LJJ, NV, PI and JMM. All authors were involved in writing and approval of the manuscript.

\section{Acknowledgements:Not applicable}

\section{References}

1. Torre LA, Bray F, Siegel RL, Ferlay J, Lortet-Tieulent J, Jemal A. Global cancer statistics, 2012. CA: a cancer journal for clinicians. 2015;65(2):87-108.

2. Abida W, Bajorin DF, Rosenberg JE. First-line treatment and prognostic factors of metastatic bladder cancer for platinum-eligible patients. Hematology/oncology clinics of North America. 2015;29(2):319-28. 
3. Knowles MA, Hurst CD. Molecular biology of bladder cancer: new insights into pathogenesis and clinical diversity. Nature Reviews Cancer. 2015;15(1):25-41.

4. Krook MA, Reeser JW, Ernst G, Barker H, Wilberding M, Li G, et al. Fibroblast growth factor receptors in cancer: genetic alterations, diagnostics, therapeutic targets and mechanisms of resistance. $\mathrm{Br} \mathrm{J}$ Cancer. 2020.

5. Billerey C, Chopin D, Aubriot-Lorton M-H, Ricol D, Gil Diez de Medina S, Van Rhijn B, et al. Frequent FGFR3 Mutations in Papillary Non-Invasive Bladder (pTa) Tumors. The American Journal of Pathology. 2001;158(6):1955-9.

6. Cappellen D, De Oliveira C, Ricol D, de Medina S, Bourdin J, Sastre-Garau X, et al. Frequent activating mutations of FGFR3 in human bladder and cervix carcinomas. Nature genetics. 1999;23(1):18-20.

7. Kimura T, Suzuki H, Ohashi T, Asano K, Kiyota H, Eto Y. The incidence of thanatophoric dysplasia mutations in FGFR3 gene is higher in low-grade or superficial bladder carcinomas. Cancer. 2001;92(10):2555-61.

8. Tomlinson D, Baldo O, Harnden P, Knowles M. FGFR3 protein expression and its relationship to mutation status and prognostic variables in bladder cancer. The Journal of pathology. 2007;213(1):91-8.

9. Guancial EA, Werner L, Bellmunt J, Bamias A, Choueiri TK, Ross R, et al. FGFR3 expression in primary and metastatic urothelial carcinoma of the bladder. Cancer Medicine. 2014;3(4):835-44.

10. Moss TJ, Qi Y, Xi L, Peng B, Kim TB, Ezzedine NE, et al. Comprehensive Genomic Characterization of Upper Tract Urothelial Carcinoma. Eur Urol. 2017;72(4):641-9.

11. Hernández S, López-Knowles E, Lloreta J, Kogevinas M, Amorós A, Tardón A, et al. Prospective study of FGFR3 mutations as a prognostic factor in nonmuscle invasive urothelial bladder carcinomas. Journal of Clinical Oncology. 2006;24(22):3664-71.

12. van Rhijn BWG, Vis AN, van der Kwast TH, Kirkels WJ, Radvanyi F, Ooms ECM, et al. Molecular Grading of Urothelial Cell Carcinoma With Fibroblast Growth Factor Receptor 3 and MIB-1 is Superior to Pathologic Grade for the Prediction of Clinical Outcome. Journal of Clinical Oncology. 2003;21(10):1912-21.

13. Brooks AN, Kilgour E, Smith PD. Molecular Pathways: Fibroblast Growth Factor Signaling: A New Therapeutic Opportunity in Cancer. Clinical Cancer Research. 2012;18(7):1855-62.

14. Al-Ahmadie HA, lyer G, Janakiraman M, Lin O, Heguy A, Tickoo SK, et al. Somatic mutation of fibroblast growth factor receptor-3 (FGFR3) defines a distinct morphological subtype of high-grade urothelial carcinoma. The Journal of pathology. 2011;224(2):270-9.

15. Di Martino E, L'hôte C, Kennedy W, Tomlinson D, Knowles M. Mutant fibroblast growth factor receptor 3 induces intracellular signaling and cellular transformation in a cell type-and mutation-specific manner. Oncogene. 2009;28(48):4306.

16. Williams SV, Hurst CD, Knowles MA. Oncogenic FGFR3 gene fusions in bladder cancer. Human Molecular Genetics. 2013;22(4):795-803. 
17. Wu Y-M, Su F, Kalyana-Sundaram S, Khazanov N, Ateeq B, Cao X, et al. Identification of targetable FGFR gene fusions in diverse cancers. Cancer discovery. 2013;3(6):636-47.

18. Webster MK, Donoghue DJ. Enhanced signaling and morphological transformation by a membranelocalized derivative of the fibroblast growth factor receptor 3 kinase domain. Molecular and cellular biology. 1997;17(10):5739-47.

19. Acquaviva J, He S, Zhang C, Jimenez J-P, Nagai M, Sang J, et al. FGFR3 Translocations in Bladder Cancer: Differential Sensitivity to HSP90 Inhibition Based on Drug Metabolism. Molecular Cancer Research. 2014;12(7):1042-54.

20. Guagnano V, Kauffmann A, Wöhrle S, Stamm C, Ito M, Barys L, et al. FGFR genetic alterations predict for sensitivity to NVP-BGJ398, a selective pan-FGFR inhibitor. Cancer discovery. 2012;2(12):1118-33.

21. Lamont F, Tomlinson D, Cooper PA, Shnyder SD, Chester J, Knowles M. Small molecule FGF receptor inhibitors block FGFR-dependent urothelial carcinoma growth in vitro and in vivo. British journal of cancer. 2011;104(1):75.

22. Karkera JD, Cardona GM, Bell K, Gaffney D, Portale JC, Santiago-Walker A, et al. Oncogenic Characterization and Pharmacologic Sensitivity of Activating Fibroblast Growth Factor Receptor (FGFR) Genetic Alterations to the Selective FGFR Inhibitor Erdafitinib. Mol Cancer Ther. 2017;16(8):1717-26.

23. Loriot Y, Necchi A, Park SH, Garcia-Donas J, Huddart R, Burgess E, et al. Erdafitinib in Locally Advanced or Metastatic Urothelial Carcinoma. New England Journal of Medicine. 2019;381(4):33848.

24. Pal SK, Rosenberg JE, Hoffman-Censits JH, Berger R, Quinn DI, Galsky MD, et al. Efficacy of BGJ398, a Fibroblast Growth Factor Receptor 1-3 Inhibitor, in Patients with Previously Treated Advanced Urothelial Carcinoma with FGFR3 Alterations. Cancer Discov. 2018;8(7):812-21.

25. Wang J, Mikse O, Liao RG, Li Y, Tan L, Janne PA, et al. Ligand-associated ERBB2/3 activation confers acquired resistance to FGFR inhibition in FGFR3-dependent cancer cells. Oncogene. 2015;34(17):2167-77.

26. Herrera-Abreu MT, Pearson A, Campbell J, Shnyder SD, Knowles MA, Ashworth A, et al. Parallel RNA interference screens identify EGFR activation as an escape mechanism in FGFR3-mutant cancer. Cancer Discov. 2013;3(9):1058-71.

27. Datta J, Damodaran S, Parks H, Ocrainiciuc C, Miya J, Yu L, et al. Akt Activation Mediates Acquired Resistance to Fibroblast Growth Factor Receptor Inhibitor BGJ398. Mol Cancer Ther. 2017;16(4):61424.

28. Riccardi C, Nicoletti I. Analysis of apoptosis by propidium iodide staining and flow cytometry. Nat Protoc. 2006;1(3):1458-61.

29. Nogova L, Sequist LV, Perez Garcia JM, Andre F, Delord JP, Hidalgo M, et al. Evaluation of BGJ398, a Fibroblast Growth Factor Receptor 1-3 Kinase Inhibitor, in Patients With Advanced Solid Tumors Harboring Genetic Alterations in Fibroblast Growth Factor Receptors: Results of a Global Phase I, Dose-Escalation and Dose-Expansion Study. J Clin Oncol. 2017;35(2):157-65. 
30. Hickinson DM, Klinowska T, Speake G, Vincent J, Trigwell C, Anderton J, et al. AZD8931, an equipotent, reversible inhibitor of signaling by epidermal growth factor receptor, ERBB2 (HER2), and ERBB3: a unique agent for simultaneous ERBB receptor blockade in cancer. Clin Cancer Res. 2010;16(4):1159-69.

31. Prahallad A, Sun C, Huang S, Di Nicolantonio F, Salazar R, Zecchin D, et al. Unresponsiveness of colon cancer to BRAF(V600E) inhibition through feedback activation of EGFR. Nature. 2012;483(7387):100-3.

32. Corcoran RB, Ebi H, Turke AB, Coffee EM, Nishino M, Cogdill AP, et al. EGFR-mediated re-activation of MAPK signaling contributes to insensitivity of BRAF mutant colorectal cancers to RAF inhibition with vemurafenib. Cancer Discov. 2012;2(3):227-35.

33. Pal S, Rosenberg J, Keam B. Efficacy of BGJ398, a fibroblast growth factor receptor (FGFR) 1-3 inhibitor, in patients with previously treated advanced/metastatic urothelial carcinoma with FGFR3 alterations. Journal of clinical oncology : official journal of the American Society of Clinical Oncology. 2016;34.

34. Loriot Y, Necchi A, Park SH, García-Donas J, Huddart RA, Burgess EF, et al. Erdafitinib (ERDA; JNJ42756493), a pan-fibroblast growth factor receptor (FGFR) inhibitor, in patients (pts) with metastatic or unresectable urothelial carcinoma (mUC) and FGFR alterations (FGFRa): Phase 2 continuous versus intermittent dosing. American Society of Clinical Oncology; 2018.

35. Katayama R, Shaw AT, Khan TM, Mino-Kenudson M, Solomon BJ, Halmos B, et al. Mechanisms of acquired crizotinib resistance in ALK-rearranged lung cancers. Science translational medicine. 2012;4(120):120ra17-ra17.

36. Engelman JA, Zejnullahu K, Mitsudomi T, Song Y, Hyland C, Park JO, et al. MET amplification leads to gefitinib resistance in lung cancer by activating ERBB3 signaling. Science. 2007;316(5827):1039-43.

37. Bean J, Brennan C, Shih JY, Riely G, Viale A, Wang L, et al. MET amplification occurs with or without T790M mutations in EGFR mutant lung tumors with acquired resistance to gefitinib or erlotinib. Proc Natl Acad Sci U S A. 2007;104(52):20932-7.

38. Ye X, Li Y, Stawicki S, Couto S, Eastham-Anderson J, Kallop D, et al. An anti-AxI monoclonal antibody attenuates xenograft tumor growth and enhances the effect of multiple anticancer therapies. Oncogene. 2010;29(38):5254-64.

39. Zhang Z, Lee JC, Lin L, Olivas V, Au V, LaFramboise T, et al. Activation of the AXL kinase causes resistance to EGFR-targeted therapy in lung cancer. Nat Genet. 2012;44(8):852-60.

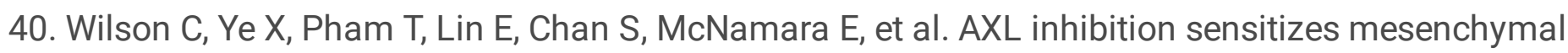
cancer cells to antimitotic drugs. Cancer Res. 2014;74(20):5878-90.

41. Wilson $C$, Ye X, Pham T, Lin E, Chan S, McNamara E, et al. AXL inhibition sensitizes mesenchymal cancer cells to antimitotic drugs. Cancer research. 2014;74(20):5878-90.

42. Antony J, Huang RY. AXL-Driven EMT State as a Targetable Conduit in Cancer. Cancer Res. 2017;77(14):3725-32. 
43. Feneyrolles C, Spenlinhauer A, Guiet L, Fauvel B, Daydé-Cazals B, Warnault P, et al. Axl kinase as a key target for oncology: focus on small molecule inhibitors. Molecular cancer therapeutics. 2014;13(9):2141-8.

44. Holland SJ, Pan A, Franci C, Hu Y, Chang B, Li W, et al. R428, a selective small molecule inhibitor of Axl kinase, blocks tumor spread and prolongs survival in models of metastatic breast cancer. Cancer research. 2010;70(4):1544-54.

45. Loges S, Torre Gjertsen B, Heuser M, Cortes JE. A first-in-patient phase I study of BGB324, a selective Axl kinase inhibitor in patients with refractory/relapsed AML and high-risk MDS. ASCO 2016. 2016.

46. Grygielewicz P, Dymek B, Bujak A, Gunerka P, Stanczak A, Lamparska-Przybysz M, et al. Epithelialmesenchymal transition confers resistance to selective FGFR inhibitors in SNU-16 gastric cancer cells. Gastric cancer : official journal of the International Gastric Cancer Association and the Japanese Gastric Cancer Association. 2016;19(1):53-62.

47. Arienti C, Pignatta S, Tesei A. Epidermal Growth Factor Receptor Family and its Role in Gastric Cancer. Front Oncol. 2019;9:1308.

48. Kunii K, Davis L, Gorenstein J, Hatch H, Yashiro M, Di Bacco A, et al. FGFR2-amplified gastric cancer cell lines require FGFR2 and Erbb3 signaling for growth and survival. Cancer Res. 2008;68(7):2340-8.

\section{Figures}

A

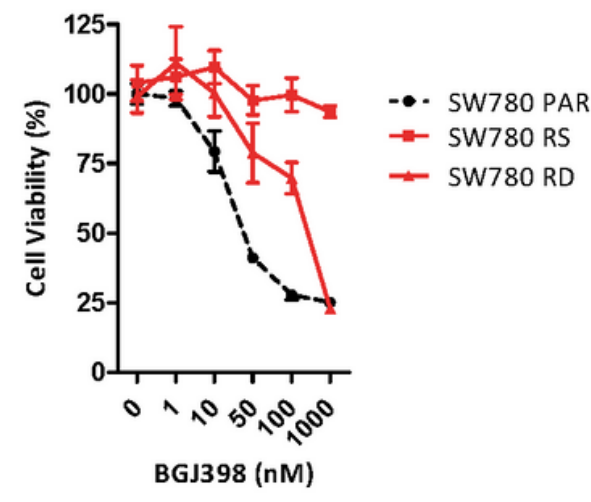

B

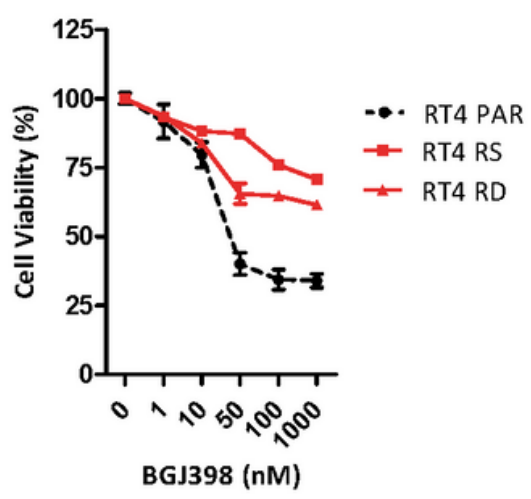

C

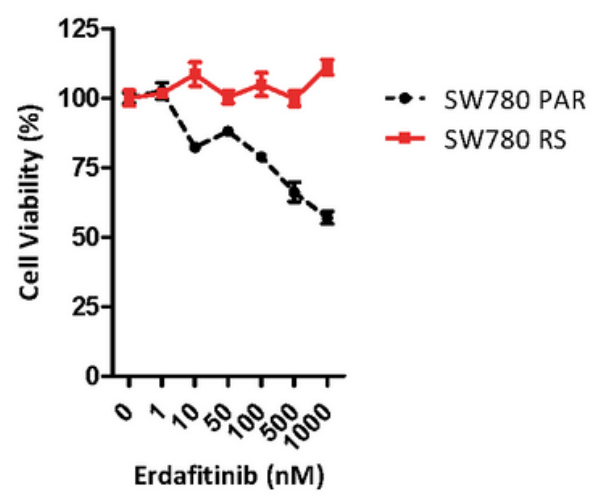

\section{Figure 1}

Sensitivity of (A) SW780 and (B) RT4 parental (PAR) and FGFR inhibitor resistant cell lines (SW780-RS, SW780-RD, RT4-RS, RT4-RD) to BGJ398. Once resistance was established, cell lines were treated with BGJ398 for 96 hours and cell viability determined using the Cell Titre-Glo assay. Values shown are mean \pm SEM of a representative experiment performed in triplicate. (C) Sensitivity of SW780-PAR and SW780RS lines to erdafitinib determined by MTS assay after 72 hours. Values shown are mean \pm SEM of a representative experiment performed in triplicate. 
A

\section{SW780 PAR}

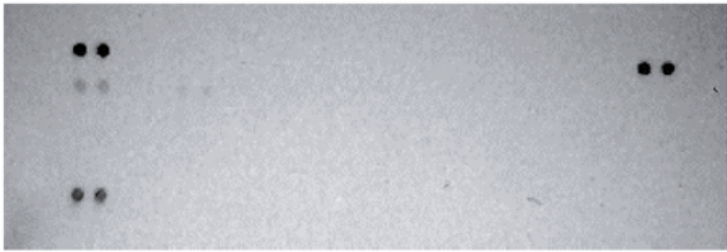

\section{SW780 RS}

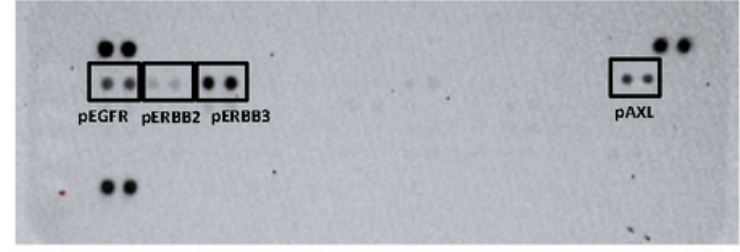

RT4 PAR

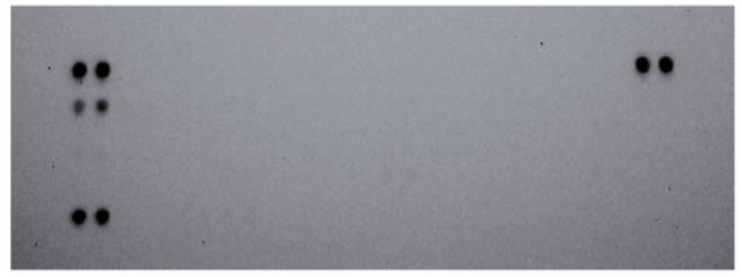

RT4 RS

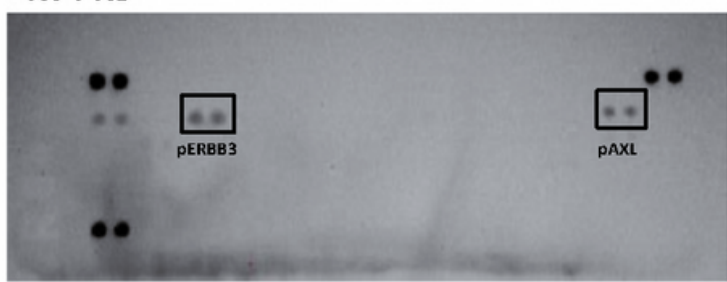

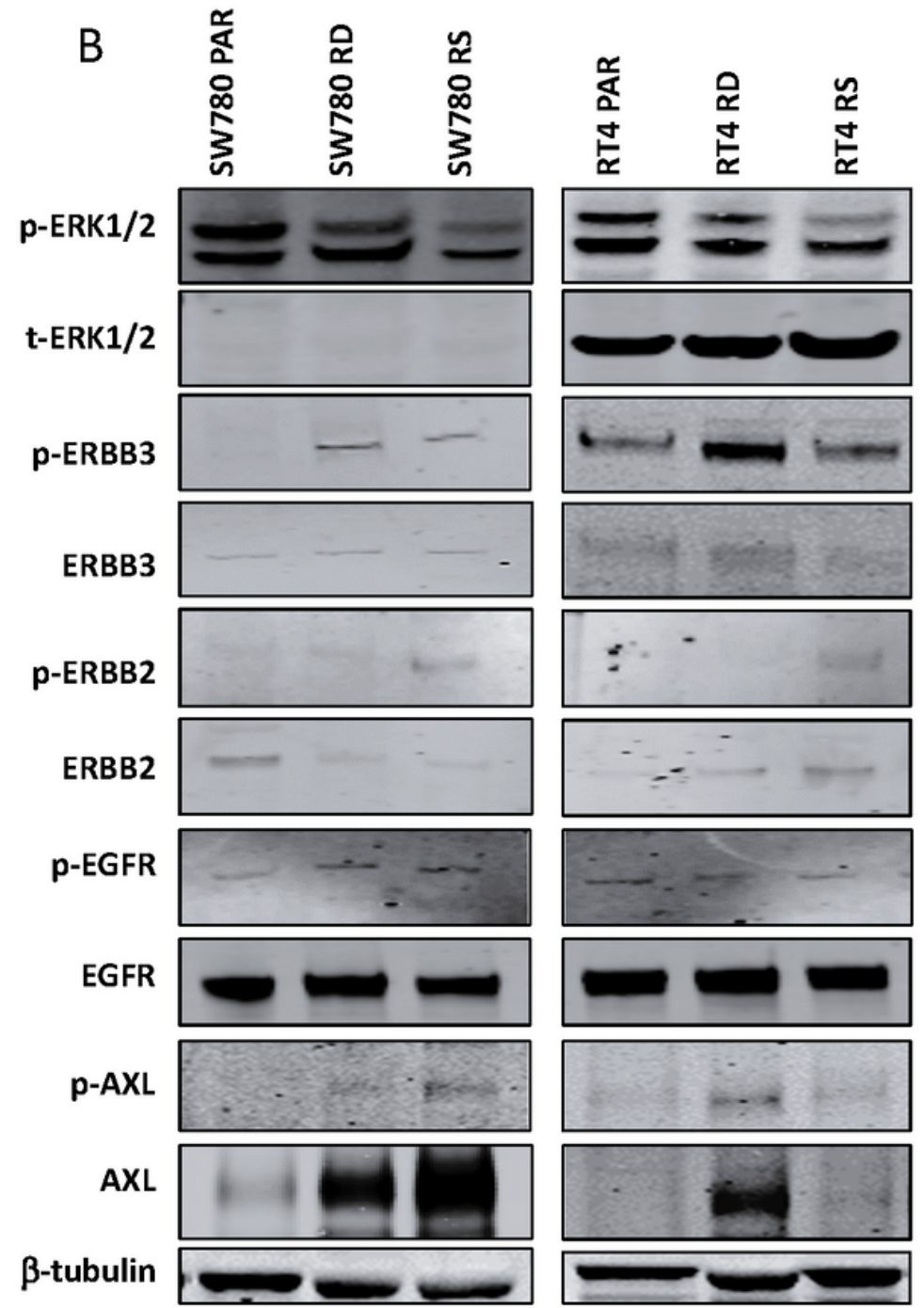

\section{Figure 2}

Activation of AXL and ERBB receptors in FGFR inhibitor-resistant bladder cancer cell lines. (A) SW780 and RT4 parental (PAR) and Resistant (RS) lines were grown in fresh medium for 24 hours without exposure to BGJ398, and cell lysates hybridized to phospho-RTK arrays. Hybridization signals at the corners serve as controls. (B) Western blot analysis confirming the increase in PAXL and ERBB family receptors in SW780 and RT4 parental (PAR) and FGFR inhibitor-resistant (RS) cell lines. Data shown are from a representative experiment. 

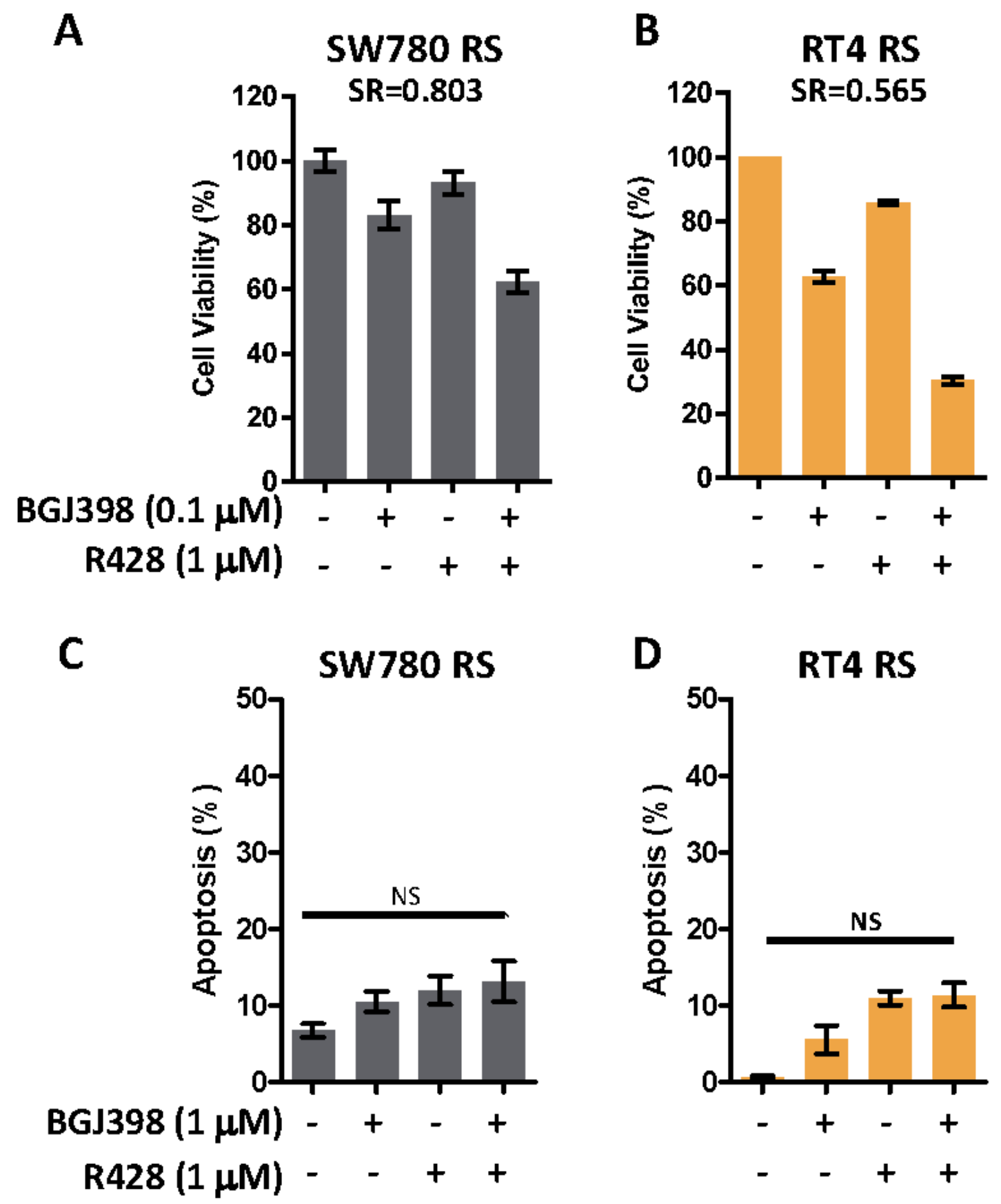

Figure 3

Effect of combinatorial inhibition of FGFR and pAXL on (A, B) cell viability and (C, D) apoptosis in cell bladder cancer cell lines with acquired resistance to FGFR inhibitors. (A, B) FGFR-inhibitor resistant (A) SW780-RS and (B) RT4-RS cell lines were treated with BGJ398 alone and in combination with the pAXL inhibitor, R428, for 72 hours and cell viability assessed using the Cell-Titer Glo assay. Values shown are mean \pm SEM of a representative experiment performed in triplicate (SR - Synergy Ratio). (C, D) FGFR 
inhibitor-resistant (C) SW780-RS and (D) RT4-RS cell lines were treated with BGJ398 alone and in combination with the PAXL inhibitor, R428, for 72 hours and apoptosis determined by PI staining and FACS analysis. Values shown are mean \pm SEM of a representative experiment performed in triplicate.
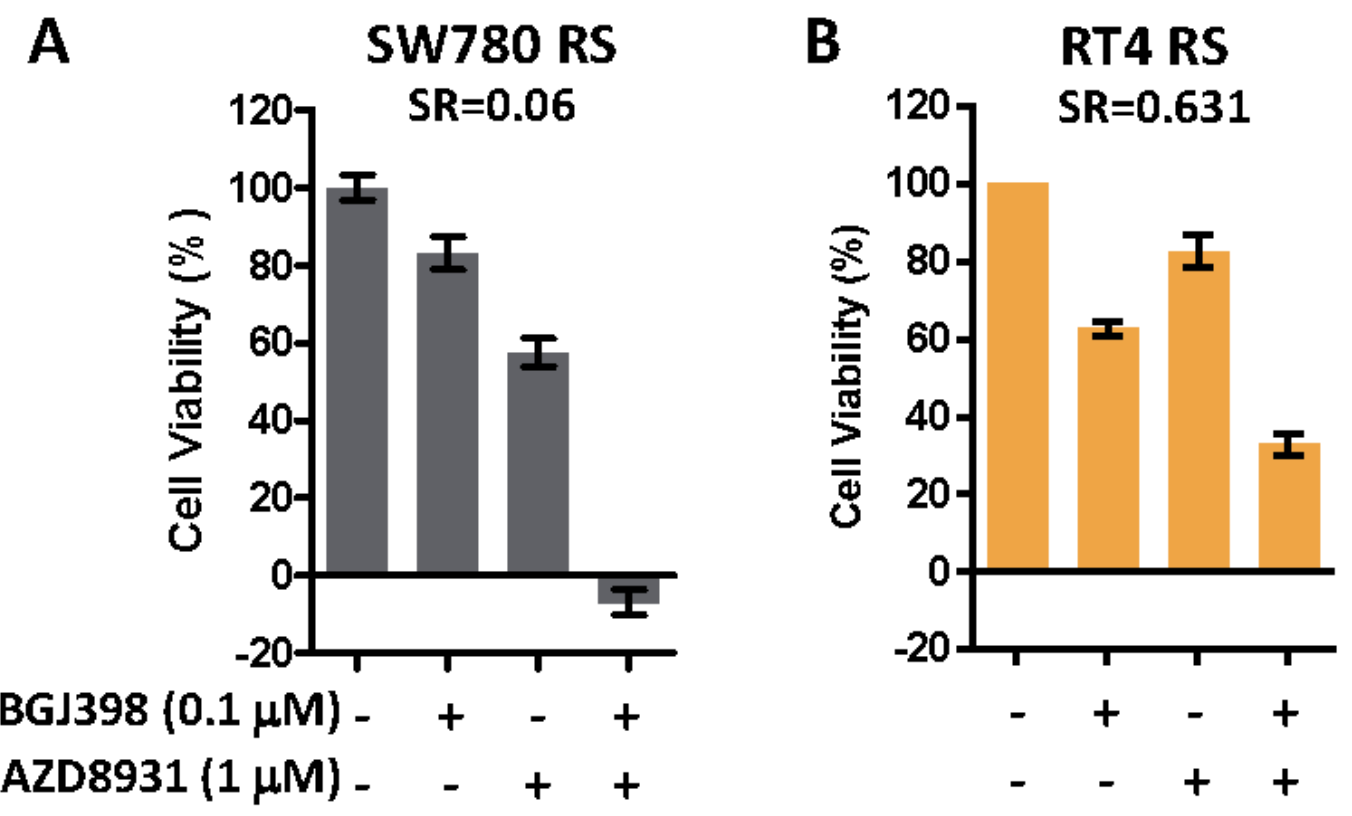

C

SW780 RS

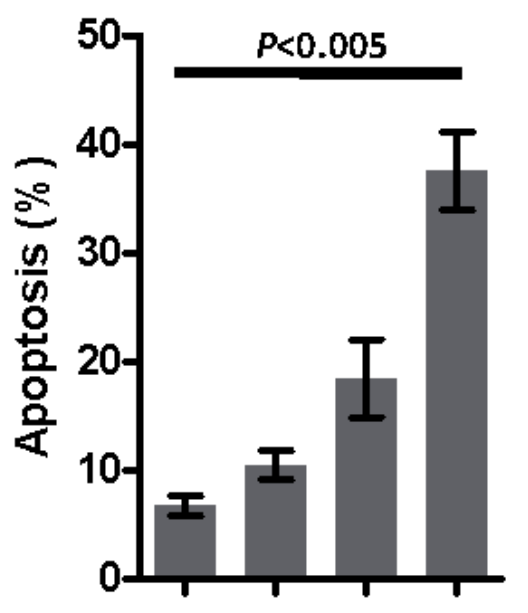

BGJ398 (1 $\mu \mathrm{M}) \quad-\quad+\quad+$ AZD8931 (1 $\mu \mathrm{M})-\quad+\quad+$

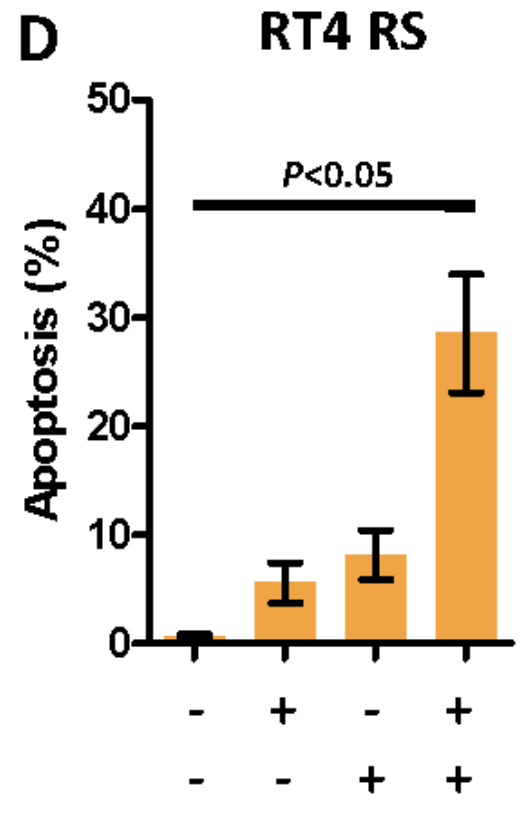

Figure 4

Effect of combinatorial inhibition of FGFR and ERBB family receptors on (A, B) cell viability and (C, D) apoptosis in cell bladder cancer cell lines with acquired resistance to FGFR inhibitors. (A, B) FGFR- 
inhibitor resistant (A) SW780-RS and (B) RT4-RS cell lines were treated with BGJ398 alone and in combination with the pan-ERBB inhibitor, AZD8931, for 72 hours and cell viability assessed using the CellTiter Glo assay. Values shown are mean \pm SEM of a representative experiment performed in triplicate (SR - Synergy Ratio). (C, D) FGFR inhibitor-resistant (C) SW780-RS and (D) RT4-RS cell lines were treated with BGJ398 alone and in combination with the pan-ERBB inhibitor, AZD8931, for 72 hours and apoptosis determined by propidium iodide staining and FACS analysis. Values shown are mean \pm SEM of a representative experiment performed in triplicate.

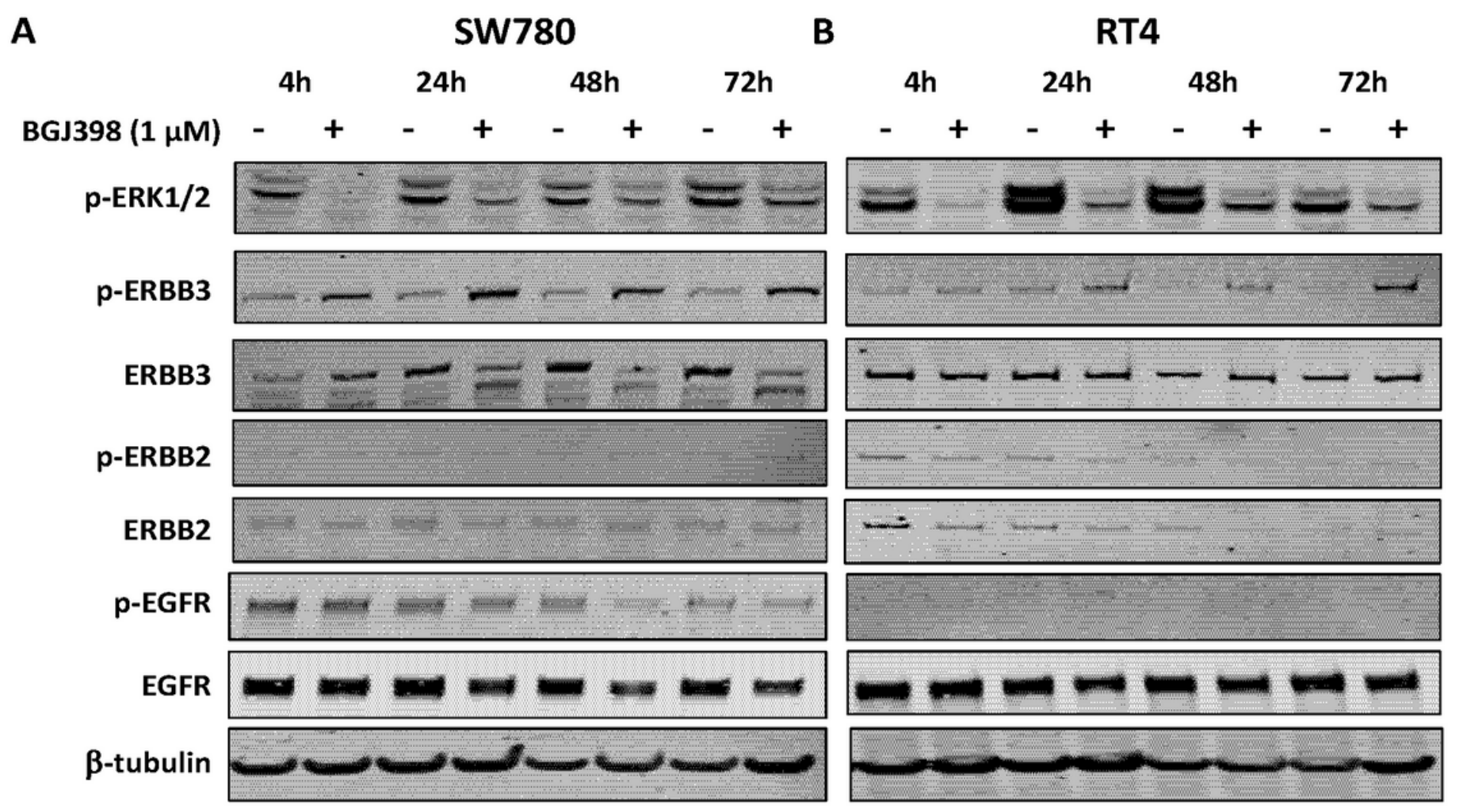

Figure 5

Effect of short term treatment with BGJ398 on pERK and ERBB family receptors. (A) SW780 and (B) RT4 parental cells were treated with BGJ398 for 4-72 hours and changes in pERK and ERBB family receptors was determined by western blot. Data shown are from a representative experiment. 


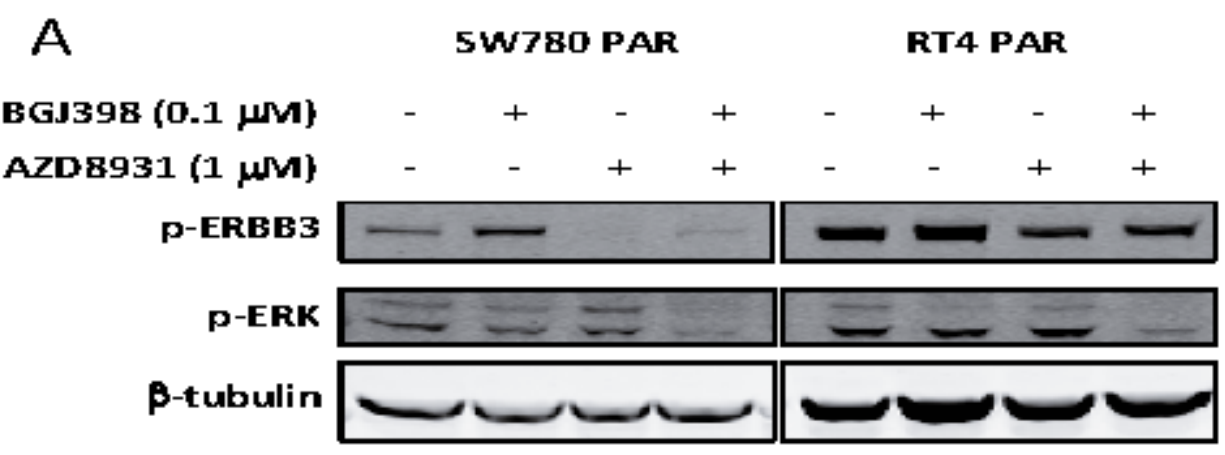

B

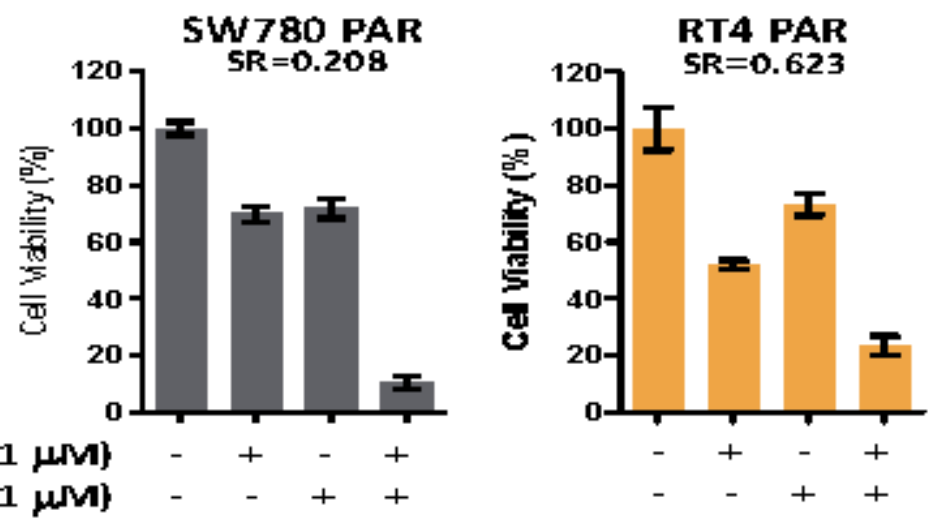

\section{Figure 6}

Effect of combinatorial inhibition of FGFR and ERBB family receptors on (A) cell signaling, and (B) cell viability in RT4 and SW780 bladder cancer cell lines. (A) Parental SW780 and RT4 cells were treated with BGJ398 (0.1 LM) or AZD8931 (1 LM), alone or in combination for 72 hours and changes in pERBB3 and pERK determined by western blot. (B) Parental SW780 and RT4 cells were treated with BGJ398 (0.1 IM) or AZD8931 (1 $\mathrm{LM})$ alone or in combination for 72 hours and cell viability determined using Cell-Titer Glo assays. Values shown are mean \pm SEM of a representative experiment performed in triplicate. 